\title{
Densely Calcified Atypical Choroid Plexus Papilloma at the Cerebellopontine Angle in an Adult
}

\author{
Masahiko Wanibuchi ${ }^{1,2}$ Russell R. Margraf ${ }^{3}$ Takanori Fukushima ${ }^{2}$
}

${ }^{1}$ Department of Neurosurgery, Sapporo Medical University School of Medicine, Sapporo, Hokkaido, Japan

${ }^{2}$ Carolina Neuroscience Institute, Raleigh, North Carolina, United States

${ }^{3}$ Raleigh Neurosurgical Clinic, Raleigh, North Carolina, United States
Address for correspondence Masahiko Wanibuchi, MD, PhD, Department of Neurosurgery, Sapporo Medical University School of Medicine, South 1, West 16, Chuo-ku, Sapporo Hokkaido 060-8543, Japan (e-mail: wanibuti@sapmed.ac.jp).

J Neurol Surg Rep 2013;74:77-80.
Abstract
Keywords
- calcification
- cerebellopontine angle
- choroid plexus papilloma
- hydrocephalus

Choroid plexus papilloma is a rare benign tumor accounting for $0.5 \%$ of all intracranial tumors. The majority of choroid plexus papillomas occur during the first 2 years of life in the ventricular system. Moreover, dense calcification of the tumor is uncommon. We report an adult case of densely calcified, atypical choroid plexus papilloma that originated from the cerebellopontine angle without connection to the forth ventricle. Because the case involves a rare combination of four features-adult, dense calcification, atypical form, and cerebellopontine angle-each characteristic associated with the choroid plexus papilloma was discussed. The finding of calcification at the cerebellopontine angle in an adult should suggest the possible presence of choroid plexus papilloma.

\section{Introduction}

Choroid plexus papilloma (CPP) is a rare benign tumor accounting for $0.5 \%$ of all intracranial tumors that is more common in childhood, consisting of 1.5 to $6.4 \%$ of pediatric intracranial tumors. ${ }^{1,2}$ The majority of CPPs occur during the first 2 years of life, with 12.5 to $20 \%$ (10 to $12 \%$ ) reported in infants under 1 year of age. ${ }^{1,3}$ There have been some reports of "calcified CPP," "CPP in an adult," "CPP in the cerebellopontine angle (CPA)," and "atypical form of CPP." ${ }^{4-7}$ However, a case combining with these four features-adult, dense calcification, atypical form, and CPA-is considered to be extremely rare; such a case has never been encountered among the 8,540 skull base tumors on which the senior author (T.F.) has operated in the past 20 years. We report an adult case of densely calcified, atypical CPP originated from the CPA without connection to the forth ventricle. Each feature of the CPP was discussed.

\section{Case Report}

A 41-year-old man presented with a chief complaint of headache, dizziness, and difficulty with gait that had been gradually worsening for 8 months. His medical and family history was unremarkable. Neurological examination revealed blurred vision, nystagmus with lateral gaze, and mild right facial weakness. Intermittent difficulty with balance and gait were also present; however, no motor weakness was observed. Computed tomography (CT) of the head without contrast revealed a high-density multilobular mass in the right CPA causing mass effect and slight ventricular enlargement (-Fig. 1). There was no evidence of adjacent bony erosion. Magnetic resonance (MR) images demonstrated a trilobed solid mass that measured $4.1 \mathrm{~cm}$ in maximum diameter (-Fig. 2). The mass was extra-axial in location and revealed isointensity on both T1-weighted images (T1-WI) and T2-weighted images (T2-WI). Homogeneous intense enhancement was identified. There was no significant flow void seen within the mass. CT and MR images did not reveal any connection between the tumor and the fourth ventricle. Gross total removal via a right lateral suboccipital approach was performed. The surface of the tumor was roughly irregular and looked grayish pink. Since the tumor was friable, gelatinous, vascular, and adherent to surrounding received

February 21, 2013

accepted

March 11, 2013

published online

May 31, 2013
DOI http://dx.doi.org/

10.1055/s-0033-1347904. ISSN 2193-6358. (c) 2013 Georg Thieme Verlag KG
Stuttgart · New York

License terms

((1) $\circledast$ 


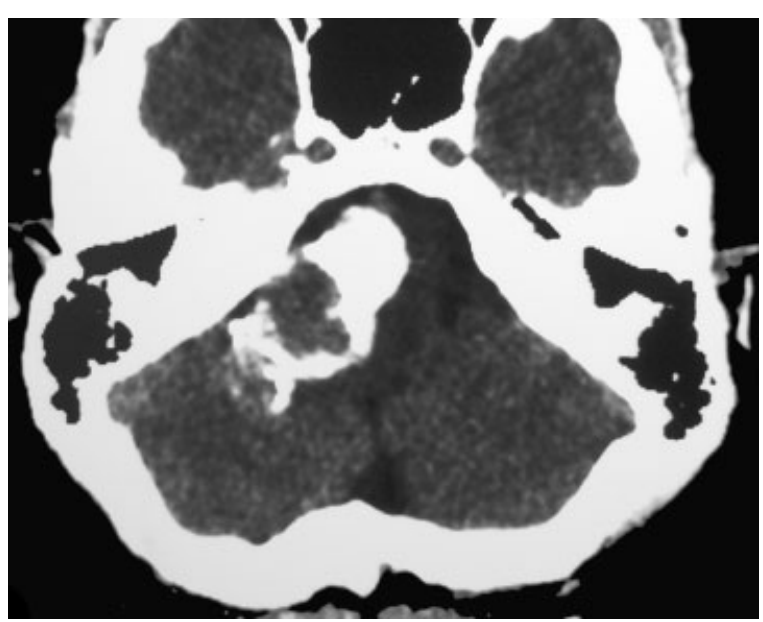

Fig. 1 Computed tomography without contrast revealed a highdensity multilobular mass in the right cerebellopontine angle. There was no evidence of adjacent bony erosion.

structures, it was hard to separate from cranial nerves. Cranial nerves VI, VII, VIII, IX, and X were enveloped in the tumor mass, which included calcification. No attachment to the clival dura was observed. Pathology reported an atypical CPP. Postoperatively the patient was alert and oriented. Language was fluent. However, slight lower cranial nerve palsies, including dysphagia, had been observed. The patient was discharged after recovery from the dysphagia by appropriate rehabilitation.
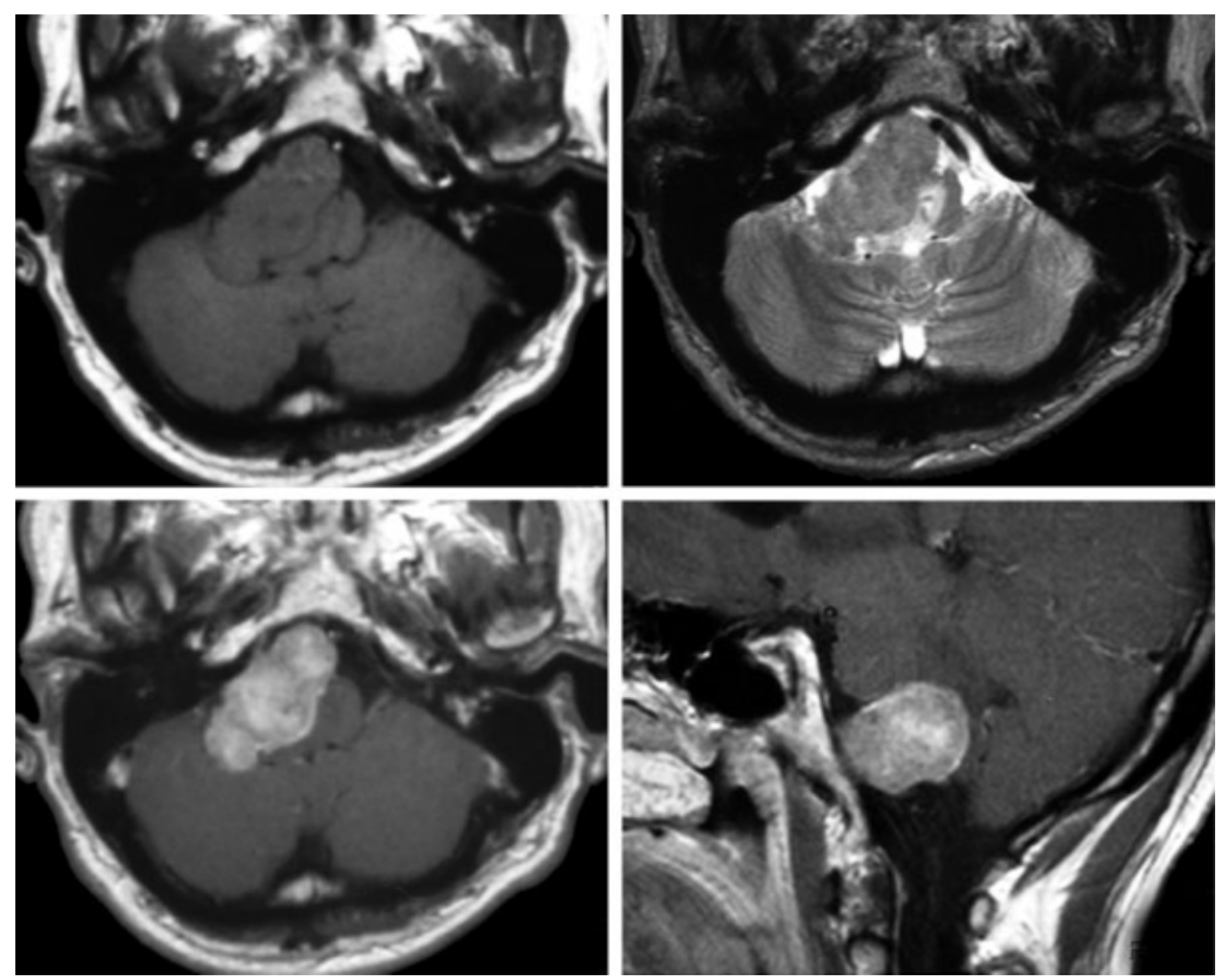

Fig. 2 Magnetic resonance images demonstrated a trilobed solid mass that was in an extra-axial location. The tumor revealed isointensity on both T1-weighted images (upper left) and T2-weighted images (upper right). Homogeneous intense enhancement was identified (axial in lower left, sagittal in lower right). There was no significant flow void seen within the mass.
CPP reported in this article primarily occurred in the CPA with close proximity to the foramen of Luschka lacking continuity of the fourth ventricle. The majority of choroid plexus tumors (CPTs) originate in the ventricular system, 43 to $67 \%$ in the lateral ventricle, 24 to $39 \%$ in the fourth ventricle, and 9.5 to $11 \%$ in the third ventricle, respectively. ${ }^{8,9}$ The common site of origin differs in each pediatric and adult group. In the pediatric age group, 60 to $80 \%$ of such tumors occur in the lateral ventricle, especially in the trigone, because the vascular stalk of the choroid plexus in the lateral ventricle is usually attached to the inferior portion of the trigone. On the other hand, most CPP in adults occurs within the fourth ventricle. ${ }^{9-11} \mathrm{~A}$ small number of the tumors have been reported to be located in extraventricular regions, the CPA, or the cerebellar hemisphere or the suprasellar cistern. ${ }^{8,10,12}$ CPP within the CPA was first described by Cushing in $1917 .^{13}$ To date, CPP within the CPA has been reported in 7 to $9 \%$ of all CPTs and is most exclusively found in adults. ${ }^{9,11,14,15}$ It is divided into two categories according to the origin of the tumor; one is primary extraventricular CPTs and the other is a result of a direct extension of fourth ventricular tumors through the foramen of Luschka. ${ }^{15}$ Because the tumor of our case revealed no connection from the fourth ventricle, it is considered to originate from small choroid tufts that normally project from each recess at the foramen Luschka into the CPA or from ectopic choroidal islets "unconnected" with the choroid plexus. ${ }^{16}$ 
The CPP of our case indicated dense calcification with ventricular enlargement. The bulbous tufts extended from the fourth ventricular choroid plexus through the foramen of Luschka have been known as the Bochdalek flower basket, which could be identified in $75 \%$ on CT and $96 \%$ on MR imaging. ${ }^{17}$ Calcification of the region was found in $38 \%$ on normal imaging anatomy. ${ }^{17}$ In pathological states, calcification of CPP was reported in $4.1 \%$ on plain skull radiographs and $23.8 \%$ on CT examinations in patients of all ages. ${ }^{8,9}$ The feature of the calcification was commonly described as a stippled or patchy configuration that may include some degrees of hemorrhage. ${ }^{8-10}$ Dense calcification was exceptionally reported by Sarkar et al. $^{2}$ The rate of hydrocephalus in association with CPP is $90 \%$ and the rate increases up to $100 \%$ in malignant CPPs. ${ }^{8}$ The cause of hydrocephalus was considered a combination of cerebrospinal fluid (CSF) overproduction (four to five times that in healthy persons), obstruction of CSF pathways by the tumor mass, impaired CSF absorption, increased protein content of CSF around the tumor, subarachnoid scarring or granulations related to recurrent bleeding from the tumor, elevated intraventricular pulse pressure, and adhesions around the exit foramina of the fourth ventricle caused by highly proteinaceous or hemorrhagic CSF. 2,10,18 The finding of tumor calcification in the CPA generally required consideration of differential diagnosis with papillary or psammomatous meningioma, melanotic schwannoma, and myxopapillary ependymoma. Among these tumors, association of ventricular enlargement with the finding of calcification might suggest the possible presence of CPTs.

Gross total removal was performed in our case of atypical CPP, although the tumor adhered to adjacent structures. In the 2007 World Health Organization Classification of Tumors of the Central Nervous System, CPTs are classified into CPP as grade I, atypical CPP as grade II, and choroid plexus carcinoma (CPC) as grade III. Among CPTs, the atypical CPP occupied in the rate of $37 \%$. Total removal of CPTs is recommended and seems to be feasible since a significant prognostic factor was relevant to the extent of tumor removal, and no definitive evidence of the beneficial effect of radiotherapy has been reported $^{6,18-20}$. However, it is not always possible because of tumor bleeding and firm adhesion to adjacent structures, especially cranial nerves. The rate of complete resection of benign CPP was $80 \%$ and the 5-year survival rate indicated is nearly $100 \%$. The majority of survivors are neurologically intact. ${ }^{19}$ Because of the good prognosis, the preservation of cranial nerve functions should be primarily considered in surgery for benign CPP. As for the atypical CPP, the rate of total removal was decreased to 50 from $63 \% .{ }^{11,21}$ Atypical CPP showed a recurrence rate of $29 \%$, which was much higher than the $6 \%$ seen in CPP. In addition, seeding within the central nervous system, spontaneous pulmonary metastasis, or rapid regrowth of atypical CPP were also reported. $^{7,22,23}$ Total or gross total removal should be advocated, especially in malignant CPTs even though the tumor encases the cranial nerves. Advertent follow-up is warranted for atypical CPP.

\section{Conclusion}

We reported an adult patient who had densely calcified atypical CPP in the CPA. The finding of calcification in the CPA should suggest the possible presence of CPP, especially if ventricular enlargement is present.

\section{References}

1 Levy ML, Goldfarb A, Hyder DJ, et al. Choroid plexus tumors in children: significance of stromal invasion. Neurosurgery 2001;48 (2):303-309

2 Sarkar C, Sharma MC, Gaikwad S, Sharma C, Singh VP. Choroid plexus papilloma: a clinicopathological study of 23 cases. Surg Neurol 1999;52(1):37-39

3 Tacconi L, Delfini R, Cantore G. Choroid plexus papillomas: consideration of a surgical series of 33 cases. Acta Neurochir (Wien) 1996;138(7):802-810

4 Jia DZ, Zhou MD, Jiang YQ Li G. Trigeminal neuralgia caused by a choroid plexus papilloma of the cerebellopontine angle: case report and review of the literature. J Int Med Res 2010;38 (1):289-292

5 Jusué-Torres I, Ortega-Zufiría JM, Tamarit-Degenhardt M, PovedaNúñez PD. [Atypical choroid plexus papilloma in adults: case report and literature review]. Neurocirugia (Astur) 2012;23 (3):116-121

6 McGirr SJ, Ebersold MJ, Scheithauer BW, Quast LM, Shaw EG. Choroid plexus papillomas: long-term follow-up results in a surgically treated series. J Neurosurg 1988;69(6):843-849

7 Tanaka K, Sasayama T, Nishihara M, et al. Rapid regrowth of an atypical choroid plexus papilloma located in the cerebellopontine angle. J Clin Neurosci 2009;16(1):121-124

8 Kendall B, Reider-Grosswasser I, Valentine A. Diagnosis of masses presenting within the ventricles on computed tomography. Neuroradiology 1983;25(1):11-22

9 Rovit RL, Schechter MM. Chodroffp Choroid plexus paillomas. Observations on radiographic diagnosis. Am J Roentgenol Radium Ther Nucl Med 1970;110(3):608-617

10 Coates TL, Hinshaw DB Jr, Peckman N, et al. Pediatric choroid plexus neoplasms: MR, CT, and pathologic correlation. Radiology 1989;173(1):81-88

11 Talacchi A, De Micheli E, Lombardo C, Turazzi S, Bricolo A. Choroid plexus papilloma of the cerebellopontine angle: a twelve patient series. Surg Neurol 1999;51(6):621-629

12 Kimura M, Takayasu M, Suzuki Y, et al. Primary choroid plexus papilloma located in the suprasellar region: case report. Neurosurgery 1992;31(3):563-566

13 Cushing H. Tumors of the Nervous Acusticus and the Syndrome of the Cerebellopontine Angle. Philadelphia WB Saunders 1917:296

14 Jackson A, Panizza BJ, Hughes D, Reid H. Primary choroid plexus papilloma of the cerebellopontine angle: magnetic resonance imaging, computed tomographic and angiographic appearances. Br J Radiol 1992;65(777):754-757

15 Piguet V, de Tribolet N. Choroid plexus papilloma of the cerebellopontine angle presenting as a subarachnoid hemorrhage: case report. Neurosurgery 1984;15(1):114-116

16 Martin N, Pierot L, Sterkers O, Mompoint D, Nahum H. Primary choroid plexus papilloma of the cerebellopontine angle: MR imaging. Neuroradiology 1990;31(6):541-543

17 Horsburgh A, Kirollos RW, Massoud TF. Bochdalek's flower basket: applied neuroimaging morphometry and variants of choroid plexus in the cerebellopontine angles. Neuroradiology 2012;54 (12):1341-1346

18 Arlt F, Trantakis C, Krupp W, et al. Cerebrospinal fluid leak after microsurgical surgery in vestibular schwannomas via retrosigmoidal craniotomy. Neurol Res 2011;33(9):947-952 
19 Ellenbogen RG, Winston KR, Kupsky WJ. Tumors of the choroid plexus in children. Neurosurgery 1989;25(3):327-335

20 Naguib MG, Chou SN, Mastri A. Radiation therapy of a choroid plexus papilloma of the cerebellopontine angle with bone involvement. Case report. J Neurosurg 1981;54(2):245-247

21 Wrede B, Hasselblatt M, Peters O, et al. Atypical choroid plexus papilloma: clinical experience in the CPT-SIOP-2000 study. J Neurooncol 2009;95(3):383-392
22 Enomoto H, Mizuno M, Katsumata T, Doi T. Intracranial metastasis of a choroid plexus papilloma originating in the cerebellopontine angle region: a case report. Surg Neurol 1991;36(1):54-58

23 Sheridan M, Besser M. Fatal pulmonary embolism by tumor during resection of a choroid plexus papilloma: case report. Neurosurgery 1994;34(5):910-912, discussion 912 